\section{Design, Fabrication and Evaluation of an Aerogel Processor CLOXS for the Astrobiology Mission Tanpopo}

\author{
Satoshi Sasaki ${ }^{1 *}$, Jun-ya Imani $^{2}$, Hajime Yano ${ }^{3}$ \\ ${ }^{1}$ School of Health Sciences, 5-23-22 Nishikamata, \\ Ohta-ku, Tokyo 144-8535, JAPAN \\ ${ }^{2}$ YUKI Precision Co., Ltd., 3-18-5 Shin-yokohama, \\ Kohoku-ku, Yokohama, 222-0033, JAPAN \\ ${ }^{3} J a p a n$ Aerospace Exploration Agency, Institute \\ of Space and Astronautical Science, 3-1-1 \\ Yoshinodai, Chuo-ku, Sagamihara, 252-5210, \\ JAPAN
}

\begin{abstract}
This paper presents a processing machine for aerogel returned to the earth after one year exposure to space at international space station. To support the astrobiology mission by processing returned sample into suitable-sized chips for biological/chemical analysis, CLOXS (Captured particles Locating, Observation and eXtracting System) was fabricated for the extraction of triangular prism-shaped chips (=Yokans) that contained collision tracks of particles that were captured in space. A novel needle was fabricated, and was mounted on the machine to cut the sides of Yokan by repeated poking motions. Typical Yokans were chosen for the processed sides roughness evaluation, and the standard deviations of the displacement were no larger than $159 \mu \mathrm{m}$, which was satisfactory by the use of $\mathbf{2 0 0 - 3 0 0 \mu m ~ d i a m e t e r ~}$ needle. 02019 Jpn. Soc. Biol. Sci. Space; doi:10.2187/ bss.33.7
\end{abstract}

\section{Introduction}

An astrobiology mission named Tanpopo is now currently operated at the Exposure Facility of Japan Experimental Module (JEM: KIBO) of the International Space Station (ISS), to examine possible interplanetary migration of microbes, and organic compounds (Yamagishi et al., 2014). One of the subthemes is about the capture of any orbiting microparticles, such as micrometeorites, space debris, and terrestrial particles carrying microbes as bioaerosols, by using blocks of silica aerogel (Tabata et al., 2011). Silica aerogel panels for bioaerosols capture were launched in April, 2015 and were placed on the Exposed Experiment Handrail Attachment Mechanism (ExHAM) in the ISS. They were successfully placed on the Exposure Facility of KIBO

Received: April 14, 2019; Accepted: July 5, 2019

*To whom correspondence should be addressed:

E-mail: sasaki@stf.teu.ac.jp
(JEM). First group of them were exposed for one year and were returned to the Earth in September, 2016 (Kawaguchi et al., 2016). Returned aerogel panels are processed into small pieces using an original machine CLOXS at ISAS clean room for the distribution to detailed analyses groups (Yano et al., 2014). The CLOXS machine processes aerogel cake into tips that is small enough to be placed in the analyzing chambers of several instruments; i.e. SEM-EDX surface observation, chemical/ biological analysis, etc., for the subsequent detailed analyses within the first 100 days after the Earth sample return (Yano et al., 2017). The CLOXS machine should, therefore, process the aerogel into desired tip size without contamination. Processing procedure should be performed under the thorough understanding of the aerogel characteristics. Similar to the laboratory analysis procedure employed in NASA's comet sample return mission Stardust (Westphal et al., 2004), needle poking motion in series was employed for the processing of aerogel panels into desired sizes. For this objective they moved needles using PC-controlled manipulators. This strategy was employed in our case with modification. Unlike the Stardust case, our Tanpopo mission employed lower density $0.01 \mathrm{~g} \mathrm{~cm}^{-3}$ aerogel. Our mission also focused in biological analysis of the captured particles. Use of single needle, instead of two, was employed to decrease contamination from the possible biological particles attached to the needle surface.

Silica aerogel is an amorphous solid with a void volume up to $99.5 \%$. Aerogel has been widely used as optical radiators for particle identification devices in high energy and nuclear physics experiments (Tabata et al., 2011). Production of this material at a wide range of densities $\left(0.01-1.2 \mathrm{~g} \mathrm{~cm}^{-3}\right.$ ) is currently possible (Tabata et al., 2011), and the one with the lowest density was used as the capture panel material in our Tanpopo mission because lower density aerogels are expected to cause less thermal damage to incident particles. Aerogels, therefore, enable intact capture of space dust particles even at hypervelocity impact of a few kilometers per second, and this factor is very important in order to carry out mineralogical and biochemical analyses in our mission. Additionally, the aerogels with a density of $0.01 \mathrm{~g} \mathrm{~cm}^{-3}$ is transparent enough (refractive index of 1.0026) (Tabata et al., 2010) to find captured micrometersize particles. The choice of a suitable solvent in the aerogel synthesis process resulted in improvements in aerogel transparency (Tabata et al., 2012). In addition, a box-framing aerogel with different inner and outer densities was designed and fabricated to resist launch vibration and to conform to an exposure attachment (Tabata et al., 2014). Finally, a mass productive fabrication method of the aerogel was optimized and as a result the flight robustness, possible biochemical contamination, and dust capture performance of the aerogel tiles were confirmed (Tabata et al., 2016).

In our study a single long glass needle was required for the processing of aerogel plates, in order to extract a larger Yokan (triangle prism-shaped aerogel chip with 
captured particles and tracks, Fig. 1-b) for the biological analysis with ca. $10 \mathrm{~mm}$ side length. Aerogel surface contamination by the human originated bacteria is minimized by cutting off the surface layer of the chip. Larger chips are, therefore, required for this purpose, and this means the need of longer ( $>30 \mathrm{~mm}$ ) needles. A novel method that enables the fabrication of longer needle using a commercial puller is required. Optimization of the needle inserting angle and control of needle depth at every poking position would enable processing to produce triangular prism. Needle manipulator is expected to move in several micrometer resolutions. Electromechanical manipulator for cell engineering is expected to meet our needs. Stages for the aerogel are desired to move also in

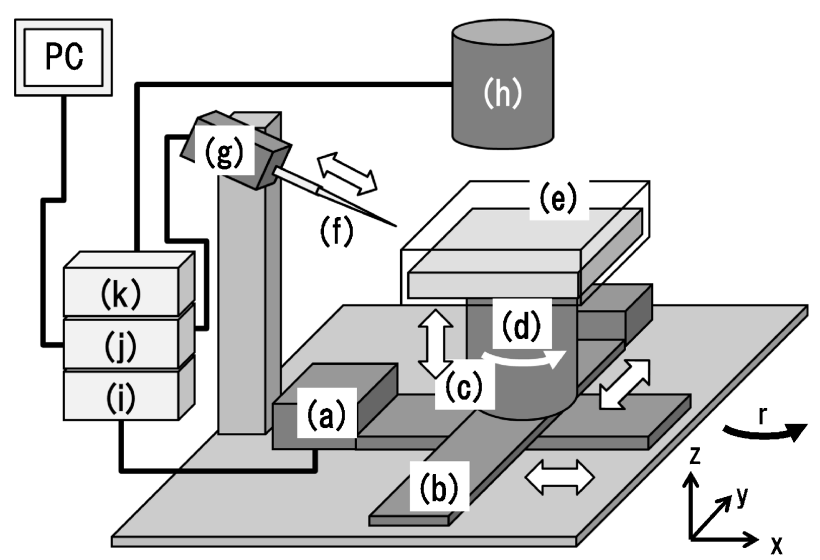

Fig. 1-a. Schematic illustration of the CLOXS system. x-axis stage (a), y-axis stage (b), z-axis stage (c), and rotation stage (d) were controlled by an original software on PC via a controller (i) (SHOT-304GS, Sigmakoki Co.,Ltd.). Aerogel panel held in an aerogel holder (e) moved along $x, y, z$, and $r$ direction, so that the relative position of the panel against the microscope (h) and the needle (f) fixed to the manipulator arm (g) was changed through the processing. Needle motion was controlled and microscopic images were recorded by the PC via manipulator (j) and a microscope imager $(\mathrm{k}) . \mathrm{x}, \mathrm{y}, \mathrm{z}$ and $\mathrm{r}$ directions were indicated to the right below of the figure.

(a)


Fig. 1-b. Schematic illustration of "Yokan", i.e. triangle prismshaped aerogel chip with captured particles and tracks. Collision of a particle (a) in space often makes a track (c) inside the aerogel panel (b). A prism-shaped chip of the aerogel, so-called "Yokan", including the whole track (sometimes with particle fragment at the end) was extracted after the processing using CLOXS. the similar spatial resolution. Both stepping motor stages and piezo electric stages were thought to satisfy this. Considering the cost performance, the former were chosen for our purpose. Main goal of the initial analysis was the processing of the aerogel quickly, safely, and with less contamination. We therefore proposed the processing machine CLOXS that can process the aerogel in a manner that satisfies the above three requirements. Objective of this paper was to fabricate CLOXS, the aerogel processing machine using an electromechanical manipulator and stepping motor stages, and to process the returned aerogel from the ISS mission into triangle prism, and finally to evaluate its size and roughness of its surface.

\section{Materials and methods}

Fabrication of the long needle

For the processing of aerogel, we used glass microneedles with metal core manufactured in our laboratory using a commercially available micropipette puller (PC-10, Narishige Co., Ltd.). Disposable capillary pipettes (ringcaps $\circledast 10 \mu$, Hirschmann Laborgerate $\mathrm{GmbH} \&$ Co. KG) were pulled at heater setting $90 \%$ of the maximum power. A metal wire (100 $\mu$ m diameter) was used as a core of the needle. Top end of the metal wire inside the capillary was placed ca. $3 \mathrm{~mm}$ above the coil heater in order to be coated by the melted glass until the tip of the needle during the pulling process.

\section{Aerogel processing}

Aerogel processing was performed for the extraction of Yokans through the repeat of following two processes; (1) repeat of aerogel poking in line using the needle poking, (2) rotation of the stage 90 degrees after pulling the needle back for $10000 \mu \mathrm{m}$ (Fig. 1). In computer program, one ID was assigned to each motion, i.e. needle pushing, pulling or stage rotation. Size of the triangle was determined as having $1200 \mu \mathrm{m}$ distance as a margin from
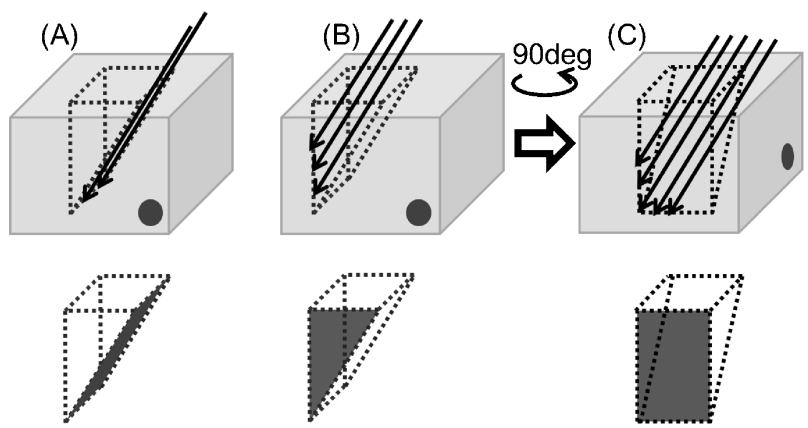

Fig. 1-c. Cutting procedure of Yokans in the aerogel processing. Relative needle poking motion against the aerogel (upper illustrations), and the Yokan sides to be cut (lower illustrations) are shown. Three sides, slope $(A)$, triangle $(B)$ and square $(C)$ were cut through the combination of needle poking (arrows) and stage horizontal translation. Both $A$ and $B$ sides were processed with the $y$ and $x$ axis translation of the stage, respectively, under the same aerogel orientation. Then the aerogel was rotated 90 degrees to cut the square side with $\mathrm{x}$ axis translation $(\mathrm{C})$. 
the entrance or track edges. This size has initial effect on the time required for the cutting. The longer it takes to cut, the higher the risk of contamination rises. We mounted the needles on commercially-available 1-axis micromanipulator system (DMA 1510 with DM system, Narishige Co., Ltd.), which were controlled by a PC (3.40 GHz with $32 \mathrm{~GB}$ memory, Diginnos, Dospara Co., Ltd.) with our original program. The needle tip was optically focused by a digital microscope (VH-5000, Keyence Co.) and all the cutting process was observed by the operators. A microneedle moved in the repeated poking motion against the aerogel. This motion was performed in line with increasing depths as the sequence proceeded. Parameters for the moving sequences were determined on the bases of least contamination by the possible falling particles. Slower needle movement was good for less damage in the cutting edge, but also indicates more possibility of attachment of the falling contaminants. Considering the schedule for management of the machine by humans, we set the needle poking motion velocity at $1250 \mu \mathrm{m} \mathrm{s}^{-1}$. Poking distance was set at $500 \mu \mathrm{m}$. The needle was set at the tip of the manipulator that moved in poking manner. An aerogel plate was placed in a holder that was fixed on a stage that moved along $x, y$, and $z$ direction. The $r$ stage also rotated along with $z$ axis. The needle orientated in the $x-z$ plane, and 50 degrees against the aerogel surface (parallel to $x-y$ plane). Cutting procedure was performed as following. The needle moved in the poking manner at 50 degrees against the aerogel surface. The needle entered into the aerogel at every poking. To cut 90 and 50 degrees planes of the Yokan, the stage moved along the $x$ and $y$ axis, respectively. Direction of the cutting was first determined by the rotation of $r$ stage, followed by the poking $-x / y$ stage movement. Poking sequence was repeated at every $200 \mu \mathrm{m}$ stage motion. After cutting all the 4 plane of the Yokan, an air tweezer was used to remove it from the plate. For the prevention of biological/ chemical contamination, aerogel processing area was arranged between the two push hoods (filtered air fans) of a super clean zone creator (KOACH, Koken Ltd.). The CLOXS system with push hoods was placed inside a clean room (CL-901, Hozan Tool Ind. Co.,Ltd.). An operator wore clean suits with mask and goggles on. After taking the air shower for $1 \mathrm{~min}$ to remove dust, he/ she entered into the clean room. Aerosol density was measured for at least 3 times using a particle counter (ABACUS-301, Artisan Technology Group), and aerogel holder cover was opened only when the 0.5 micrometer particle flux was below the lower limit of detection.

\section{Yokan evaluation}

Microscopic observation of the extracted Yokans was performed and their images were binarized using an image processing application. Edge of the Yokan was figured out using the same application, and the data was converted into the text data that was finally processed by the commercially available spreadsheet application. Displacements of the edges of the Yokan were statistically evaluated as the standard deviations. Three angles of the cross section triangle were measured, and their sizes were compared with the programmed ones. Image processing was performed using an image processing program called ImageJ (a public domain, Java-based PC application developed at the National Institutes of Health) (Schneider et al., 2012). Image of a Yokan was processed into a black and white (binary) image using "Make Binary" command of the application found in "Process" tab. The image only with the Yokan edge was then obtained by the "Find Edges" command. Finally, the edge image was saved as "Text Image" and the file was opened using Microsoft Excel. Using the "MATCH()" function, that allows us to find the relative position of a value in a list in Excel, displacements of the edge data from lines parallel to the surface, vertical side, and slope side were measured.

\section{Results}

\section{Fabrication of the long needle}

A metal cored needle with ca. $30 \mathrm{~mm}$ length was fabricated ca. $10 \mathrm{~s}$ after the heating. Its diameter was ca. $300 \mu \mathrm{m}$ at the neck, and near the tip $200 \mu \mathrm{m}$. The very tip of the needle the curvature radius was too small to be evaluated by the optical microscope. The metal wire was not continuously filled inside the glass, but intermittently enclosed inside the needle (Fig. 2). Without the metal wire core, the needle often was not straight and its length did not exceed $30 \mathrm{~mm}$.

\section{Aerogel processing}

For the extraction of over 70 Yokans from the firstyear returned 12 aerogels, one needle was used for every aerogel. One needle was good and flexible enough for the processing of several Yokans without breaking; it endured several thousands of poking. Small Yokan with one side length of several mm required ca. $4 \mathrm{~h}$, and large one with several tens $\mathrm{mm} 2$ days maximum. The size of the triangle (Yokan) determines the cutting time and should initially determine the risk of contamination. Total poking steps for cutting, although not linearly related to the cutting time, was examined in relation to the length of the track. Through this relationship we should see the



Fig. 2. Typical image of a needle. Numbers of the scale are in centimeters. 


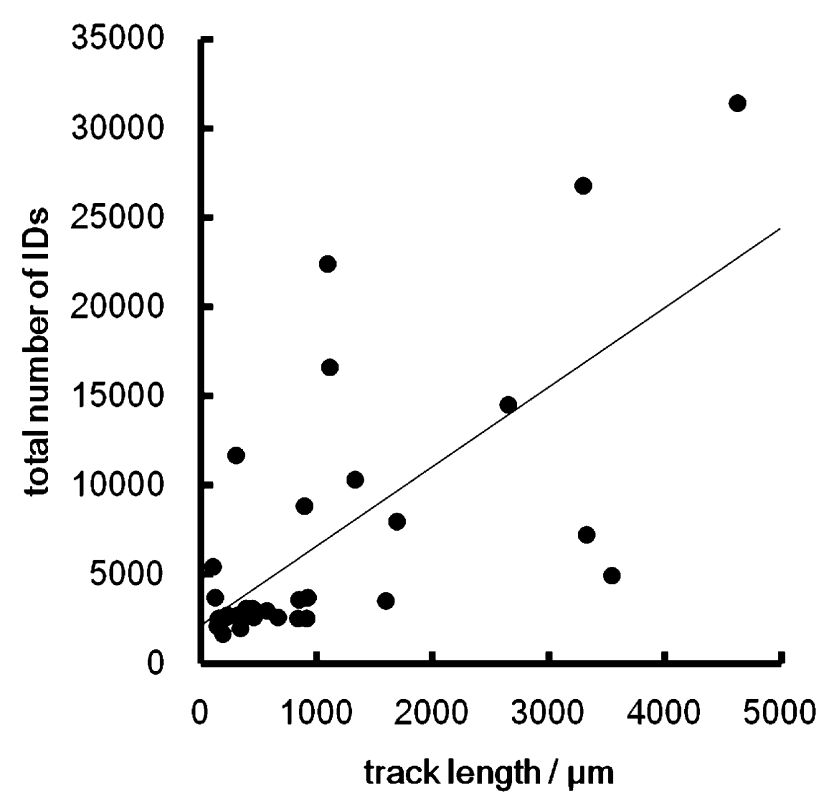

Fig. 3. Relationship between the track length and the total number of IDs. Solid line indicates the linear fitting of the data. Coefficient of determination was 0.473 .

validity of the cutting size determination. Fig. 3 shows the relationship between the track length or entrance size and the total poking steps for 33 extracted Yokans in the Tanpopo project. As is clear from the figure, total poking IDs varied even for the tracks with similar length. Considering the incident angle of the needle against the aerogel surface being 40 degrees, maximum poking distance of the needle before reaching the bottom of the aerogel can be calculated. After the return of the aerogel to the Earth, the thickness decreased down to ca. $14 \mathrm{~mm}$, so the needle tip should reach the bottom after $18.4 \mathrm{~mm}$ of insertion. For the tracks with $>10 \mathrm{~mm}$ length, total IDs, as well as total cutting time or contamination risk, should be determined by the maximal diameter of the track. So far, total IDs of ca. 31400 was the largest and unlike other tracks the entrance hole was easily visible by the naked eyes.

\section{Yokan evaluation}

Standard deviation, which represents the variety in the displacements from the mean value, was calculated to evaluate the flatness of the processed Yokans. The displacements should, theoretically, be zero when the surface is completely flat. The evaluation was performed using the microscopic images of Yokans. Fig. 4 shows the typical example of Yokan image before $(A)$ and after (B) the image processing. During the cutting process, a needle with more than $200 \mu \mathrm{m}$ diameter stuck every $200 \mu \mathrm{m}$ of the aerogel surface. When the needle was inserted at the deepest position, the aerogel surface accepted $300 \mu \mathrm{m}$ diameter part of the needle. Burrs with maximum $150 \mu \mathrm{m}$ length could, theoretically, remain at the cut edge of the aerogel. Microscopic cross section images of four Yokans were evaluated. Surface width (set/

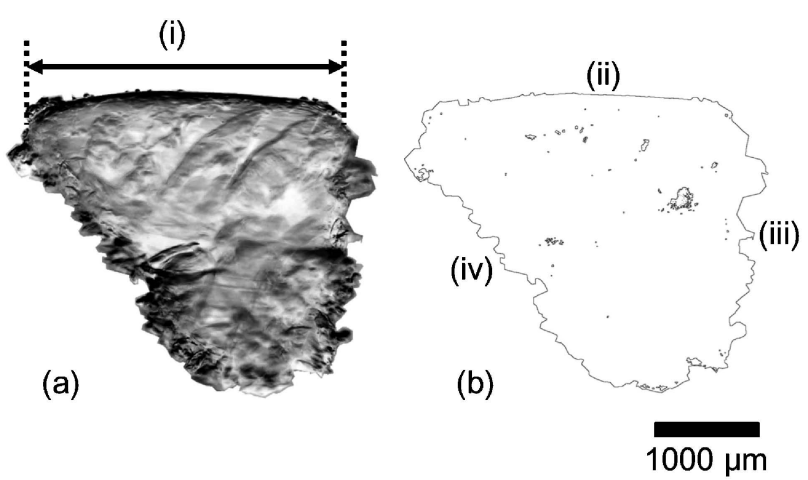

Fig. 4. Typical image of a Yokan with artificial white background (a), and its sampled edge image (b). Width of surface side (i), surface (ii), vertical side (iii), and slope side (iv) are shown. Processing of the image (a) to (b) was performed using the ImageJ application.

Table 1. Size and roughness evaluation of 4 typical Yokans. Measurement of width of surface side was performed manually by the use of scale against the microscopic images.

\begin{tabular}{c|c|c|c|c|c}
\hline $\begin{array}{c}\text { Yokan } \\
\text { ID }\end{array}$ & \multicolumn{2}{|c|}{$\begin{array}{c}\text { width of surface side/ } \\
\mu m\end{array}$} & \multicolumn{3}{|c}{ standard deviation/ $\mu \mathrm{m}$} \\
\hline & set & measured & surface & $\begin{array}{c}\text { vertical } \\
\text { side }\end{array}$ & $\begin{array}{c}\text { slope } \\
\text { side }\end{array}$ \\
\hline F1_479 & 3400 & $3400-3600$ & 31 & 80.4 & 80 \\
\hline F1_328 & 3200 & $2900-3000$ & 51.7 & 130 & 71.1 \\
\hline L1_216 & 10000 & $9800-10600$ & 87.5 & 105 & 68.2 \\
\hline P1_320 & 8400 & $8300-8700$ & 82.7 & 159 & 86.1 \\
\hline
\end{tabular}

measured values) of Yokans was also compared. Vertical and slope sides against the surface were examined, together with the surface. As is clear from the results shown in table 1, differences between the set and measured width were within $200 \mu \mathrm{m}$. Surface roughness defined as the standard deviation was largest with the vertical side. 3 out of 4 Yokans showed lager value with slope sides compared to surface. The standard deviation values were within the range of 68-159 $\mu \mathrm{m}$. Considering the diameter of the needle to be ca. $200 \mu \mathrm{m}$, this value was unexpectedly good considering the theoretically expected burr size.

\section{Discussion}

As mentioned, size the triangle to be cut was determined by the $1200 \mu \mathrm{m}$ margin from the edge of the track. This should result in the difference in total IDs according to the shape of the tracks, ie., carrot, burst, or rugby ball (Burchell et al., 2008). For the extraction of more than 70 Yokans out of 12 returned aerogels, we applied the above mentioned protocols, and the processed Yokans were handled to the detailed analysis (biological/chemical) teams. Through the ongoing elemental analysis or biomolecule detection, no critical contamination problem due to the processing was reported. Notably, during the processing, small aerogel fragments adhered to the needle and were blown off by the wind from the push hood. Particle counter value 
exceeded the lower limit of detection with these fragments. In this sense, transition of the aerogel fragments to the portion of analysis would be the concerned matter. Falling lubricant from the manipulator might also be the possible contaminant. The damage to the surrounding tracks after the extraction of neighbor Yokan was minimized. Precision cutting of aerogel on the micron scale was reported as a difficult challenge for the $0.03 \mathrm{~g} \mathrm{~cm}^{-3}$ aerogel (Westphal et al., 2002). Our case with $0.01 \mathrm{~g} \mathrm{~cm}^{-3}$ aerogel was imagined to be more difficult but as a result successfully processed. Aerogel processing using a commercial laser processor resulted in the black burnt at the cut surface. This method was suspected both to damage and to contaminate the keystone track during the process. Cutting the aerogel manually using the razor blade by a well-trained person was well-controlled and reliable, but too elaborate and not suitable for the mass processing of the hundreds of keystone tracks. As a conclusion the use of CLOXS with the procedure we reported was suitable, not to say optimal, for the Yokan extraction from $0.01 \mathrm{~g} \mathrm{~cm}^{-3}$ aerogels.

\section{Acknowledgment}

This work was supported by the Astrobiology Center Program of National Institutes of Natural Sciences (NINS).

\section{Conflicts of Interest}

The authors declare no competing interests.

\section{References}

Burchell, M. J., Fairey, S. A. J., Wozniakiewicz, P., Brownlee, D. E., Horz, F., Kearsley, A. T., See, T. H., Tsou P., Westphal, A., Green, S. F., Trigo-Rodriguez, J. M., and Dominguez G. (2008) Characteristics of cometary dust tracks in Stardust aerogel and laboratory calibrations. Meteor. Planet. Sci. 43, 23-40.

Kawaguchi, Y., Yokobori, S., Hashimoto, H., Yano, H., Tabata, M., Kawai, H., and Yamagishi, A. (2016) Investigation of the Interplanetary Transfer of Microbes in the Tanpopo Mission at the Exposed Facility of the International Space Station. Astrobiol., 16, 363-376.

Schneider, C. A., Rasband, W. S., and Eliceiri, K. W. (2012) NIH Image to ImageJ: 25 years of image analysis, Nature Methods, 9, 671-675.

Tabata, M., Kawai, H., Yano, H., Imai, E., Hashimoto, H., Yokobori, S., Yamagishi, A. (2016) Ultralow-density double-layer silica aerogel fabrication for the intact capture of cosmic dust in low-Earth orbits. J. Sol-Gel Sci. Technol., 77, 325-334.

Tabata, M., Imai, E., Yano, H., Hashimoto, H., Kawai, H., Kawaguchi, Y., Kobayashi, K., Mita, H., Okudaira, K., Sasaki, S., Yabuta, H., Yokobori, S., and Yamagishi, A. (2014) Design of a Silica-aerogel-based Cosmic Dust Collector for the Tanpopo Mission Aboard the
International Space Station. Trans. Jpn. Soc. Aeronaut. Space Sci., 12, 29-34.

Tabata, M., Adachi, I., Kawai, H., Sumiyoshi, T., and Yokogawa, H. (2012) Hydrophobic silica aerogel production at KEK. Spectrometer Detector Assoc. Equip., 668, 64-70.

Tabata, M., Kawaguchi, Y., Yokobori, S., Kawai, H., Takahashi, J., Yano, H., and Yamagishi, A. (2011) Tanpopo Cosmic Dust Collector: Silica Aerogel Production and Bacterial DNA Contamination Analysis. Biol. Sci. Space., 25, 7-12.

Tabata, M., Adachi, I., Ishii, Y., Kawai, H., Sumiyoshi, T., and Yokogawa, H. (2010) Development of transparent silica aerogel over a wide range of densities. Nucl. Instrum. Methods Phys. Res. A., 623, 339-341.

Westphal, J., Snead, C., Butterworth, A., Graham, G. A., Bradley, J. P., Bajt, S., Grant, P. G., Bench, G., Brennan, S., and Pianetta, P., (2004) Aerogel keystones: Extraction of complete hypervelocity impact events from aerogel collectors. Meteor. Planet. Sci., 39, 1375-1386.

Westphal, J., Snead, C. J., Borg, J., Quirico, E., Raynal, P. I., Zolensky, M. E., Ferrini, G., Colangeli, L., and Palumbo. P., (2002) Small hypervelocity particles captured in aerogel collectors: Location, extraction, handling, and storage. Meteor. Planet. Sci., 37, 855865.

Yamagishi, A., Yokobori, S., Hashimoto, H., Yano, H., Higashide, M. Tabata, Imai, E., Yabuta, H., Kobayashi, K., and Kawai, H., (2014) Tanpopo: Astrobiology Exposure and Micrometeoroid Capture Experiments? Proposed Experiments at the Exposure Facility of ISSJEM. Trans. Jpn. Soc. Aeronaut. Space Sci., 12, 4955.

Yano, H., Sasaki, S., Imani, J., Horikawa, D., Arai, K., Fujishima, K., Hashimoto, H., Higashide, M., Imai, E., Ishibashi, Y., Kawaguchi, Y., Kawai, H., Kebukawa, Y., Kobayashi, K., Kobunai, K., Kodaira, S., Kurosu, Y., Mita, H., Oda, Y., Okudaira, K., Ozawa, T., Tabata, M., Takizawa, N., Tomita, M., Tsuchiyama, A., Uchihori, Y., Yabuta, H., Yaguchi, Y., Yokobori, S., Yamagishi, A., and Tanpopo Project Team. (2017) In-Orbit Operation and Initial Sample Analysis and Curation Results for the First Year Collection Samples of the Tanpopo Project. 48th Lunar and Planetary Science Conference, The Woodlands, Texas, March 20-24.

Yano, H., Yamagishi, A., Hashimoto, H., Yokobori, S., Kobayashi, K., Yabuta, H., Mita, H., Tabata, M., Kawai, H., Higashide, M., Okudaira, K.,Sasaki, S., Imai, E., Kawaguchi, Y., Uchibori, Y., Kodaira, S., and the Tanpopo Project Team. (2014) Tanpopo experiment for astrobiology exposure and micrometeoroid capture onboard the ISS-JEM exposed facility. 45th Lunar and Planetary Science Conference, The Woodlands, Texas, March 17-21. 